\title{
Hukuhara's Topological Degree for non Compact Valued Multifunctions
}

\author{
By
}

Francesco S. De Blasi* and Pando Gr. Georgiev**

\begin{abstract}
We present a direct construction of a topological degree for multivalued vector fields $I-F$ in a Banach space, where $F$ takes closed, bounded, convex (or non convex) values and the set-valued range of $F$ is precompact in the Pompeiu-Hausdorff metric. Some useful properties of our topological degree are established. Applications to fixed point theory including a Borsuk's type result are considered.
\end{abstract}

\section{$\S 1$. Introduction}

Let $\mathbb{E}$ be a real Banach space and let $F$ be a multifunction defined on a non empty open bounded subset of $\mathbb{E}$, whose values are non empty subsets of $\mathbb{E}$. If $I$ stands for the identity mapping in $\mathbb{E}$, the multifunction $I-F$ will be called a multivalued vector field.

A topological degree theory for multivalued vector fields $I-F$, when $F$ is a Pompeiu-Hausdorff upper semicontinuous ( $h$-u.s.c.) multifunction with non empty compact convex values, was developed by Hukuhara [16] in a classical

Communicated by Y. Takahashi. Received August 9, 1999. Revised November 14, 2000 and January 17, 2002.

2000 Mathematics Subject Classification(s): 47H10, 58C06.

Key words: topological degree, multivalued mappings, fixed points.

*Department of Mathematics, University of Roma II 'Tor Vergata', Via della Ricerca Scientifica, 00133 Roma, Italy.

e-mail: deblasi@mat.uniroma2.it

** Department of Mathematics and Informatics, Sofia University 'St. Kl. Ohridski', 5 James Bourchier Blvd., 1126 Sofia, Bulgaria.

Current address: Laboratory for Advanced Brain Signal Processing, Brain Science Institute, The Institute of Physical and Chemical Research (RIKEN), 2-1, Hirosawa, Wakoshi, Saitama 351-0198, Japan.

e-mail: georgiev@bsp.brain.riken.go.jp 
paper published in 1967. Hukuhara's topological degree retains the fundamental properties of Leray-Schauder topological degree [20] and, like the latter, has several applications (see Hu and Papageorgiou [15], Lloyd [21], Ma [22]). In particular, it permits to give alternative proofs of fixed point theorems for multifunctions of the type of Kakutani [18] and Ky Fan [7]. Significant developments of Hukuhara's theory can be found among others, in Cellina and Lasota [3], Lasry and Robert [19], Ma [22], Fitzpatrick and Petryshyn [8], [24] and Borisovitch, Gelman, Myshkis and Obukhovskii [2].

The problem of extending the Hukuhara topological degree to multivalued vector fields $I-F$, in which $F$ takes non empty closed bounded and convex values, was considered by De Blasi and Myjak [6]. However the notion of topological degree introduced in [6] was too weak, sufficient only to prove existence of almost fixed points. Recently the problem has been considered, in a general setting, by Dawidowicz [4] who has introduced a more appropriate notion of topological degree, which is useful also in fixed point theory. The approach of Dawidowicz is, to a certain extent, not elementary, since it relies on advanced techniques of homology theory along a line of research which goes back to the fundamental contributions of Granas [13], Geba and Granas [9] and Górniewicz [10].

The aim of this paper is to present an elementary and direct construction of a topological degree for multivalued vector fields $I-F$, where $F$ takes non empty closed bounded convex, or non convex, values. For a multifunction $F$ the usual notion of compactness is too restrictive for our needs, and thus we will replace it by the $h$-compactness of $F$, a notion introduced in [6], which essentially requires that the set-valued range of $F$ be precompact in the Pompeiu-Hausdorff metric $h$. In our approach a fundamental role is played by approximation techniques very much as in Hukuhara [16] and Cellina and Lasota [3]. Further developments in this spirit can be found in Górniewicz, Granas and Kryszewski [11], Górniewicz and Lassonde [12], Borisovitch, Gelman, Myshkis and Obukhovskii [2] as well as in the comprehensive monograph on multifunctions by $\mathrm{Hu}$ and Papageorgiou [15].

The paper consists of five sections. Section 2 contains notation and preliminaries. In Section 3 we define a topological degree for multivalued vector fields $I-F$, in which $F$ is a regular multifunction (see Definition 3.2). Our degree reduces to that of Hukuhara, when $F$ is a $h$-u.s.c. compact multifunction taking non empty compact convex values. A few useful properties of our topological degree are established in Section 4. Applications to fixed point theory including a Borsuk's type result are contained in Section 5. 


\section{§2. Notation and Terminology}

Throughout this paper $M$ denotes a metric space, $\mathbb{E}$ a real Banach space with norm $\|\cdot\|, I$ is the identity map in $\mathbb{E}$. Furthermore, $2^{\mathbb{E}}$ will denote the family of all non empty subsets of $\mathbb{E}$, and

$$
\begin{aligned}
& \mathcal{H}(\mathbb{E})=\left\{X \in 2^{\mathbb{E}}: X \text { is compact }\right\}, \\
& \mathcal{K}(\mathbb{E})=\left\{X \in 2^{\mathbb{E}}: X \text { is compact convex }\right\}, \\
& \mathcal{C}(\mathbb{E})=\left\{X \in 2^{\mathbb{E}}: X \text { is closed bounded convex }\right\} \\
& \mathcal{B}(\mathbb{E})=\left\{X \in 2^{\mathbb{E}}: X \text { is closed bounded }\right\} .
\end{aligned}
$$

For $a \in \mathbb{E}$ and $X \in 2^{\mathbb{E}}$, we set $d(a, X)=\inf _{x \in X}\|a-x\|$. Each space $\mathcal{H}(\mathbb{E})$, $\mathcal{K}(\mathbb{E}), \mathcal{C}(\mathbb{E}), \mathcal{B}(\mathbb{E})$ is endowed with the Pompeiu-Hausdorff metric:

$$
h(X, Y)=\max \{e(X, Y), e(Y, X)\},
$$

where $e(X, Y)=\sup _{x \in X} d(x, Y)$ and $e(Y, X)=\sup _{y \in Y} d(y, X)$.

Remark 2.1. Under the Pompeiu-Hausdorff metric $h$ each space $\mathcal{K}(\mathbb{E})$, $\mathcal{C}(\mathbb{E}), \mathcal{B}(\mathbb{E})$ is complete. Furthermore, $\mathcal{H}(\mathbb{E}), \mathcal{K}(\mathbb{E})$ and $\mathcal{C}(\mathbb{E})$ are closed subsets of $\mathcal{B}(\mathbb{E})$.

For $X \subset M$, by $\bar{X}$ or $c l_{M} X$ we mean the closure of $X$ in $M$, and by $\partial X$ the boundary of $X$. Moreover, $U_{M}(a, r)$ is the open ball in $M$ with center $a$ and radius $r$. In $\mathbb{E}$ instead of $U_{\mathbb{E}}(a, r)$ and $U_{\mathbb{E}}(0,1)$ we write, for brevity, $U(a, r)$ and $U$.

The convex hull and closed convex hull of $X \subset \mathbb{E}$ are denoted by $c o X$ and $\overline{c o} X$, respectively.

Let $F$ be a map which associates with each $x \in M$ a non empty subset $F(x)$ of $\mathbb{E}$. When, for each $x \in M, F(x)$ is a subset of $\mathbb{E}$ in a family, say $\mathcal{F}(\mathbb{E})$, of subsets of $\mathbb{E}$, we write (by abuse of notation) $F: M \rightarrow \mathcal{F}(\mathbb{E})$, and we call $F$ a multifunction, or $\mathcal{F}(\mathbb{E})$-valued multifunction.

The $\mathbb{E}$-range $\mathcal{R}_{\mathbb{E}}(F)$ of $F$ and the $\mathcal{F}(\mathbb{E})$-range $\mathcal{R}_{\mathcal{F}(\mathbb{E})}(F)$ of $F$ are defined by

$$
\begin{aligned}
\mathcal{R}_{\mathbb{E}}(F) & =\{y \in E: \text { there is } x \in M \text { such that } y \in F(x)\}, \\
\mathcal{R}_{\mathcal{F}(\mathbb{E})}(F) & =\{Y \in \mathcal{F}(\mathbb{E}): \text { there is } x \in M \text { such that } Y=F(x)\} .
\end{aligned}
$$

When $F: M \rightarrow \mathbb{E}$ is single-valued, the $\mathbb{E}$-range $\mathcal{R}_{\mathbb{E}}(F)$ of $F$ is also denoted by $F(M)$. 
Definition 2.1. A multifunction $F: M \rightarrow \mathcal{B}(\mathbb{E})$ is said to be PompeiuHausdorff upper semicontinuous (resp. lower semicontinuous and continuous) if for every $x_{0} \in M$ and $\varepsilon>0$ there is $\delta>0$ such that $x \in U_{M}\left(x_{0}, \delta\right)$ implies $e\left(F(x), F\left(x_{0}\right)\right)<\varepsilon\left(\operatorname{resp} . e\left(F\left(x_{0}\right), F(x)\right)<\varepsilon\right.$ and $\left.h\left(F(x), F\left(x_{0}\right)\right)<\varepsilon\right)$.

Instead of Pompeiu-Hausdorff upper semicontinuous (resp. lower semicontinuous and continuous) we write, for convenience, $h$-u.s.c. (resp. $h$-l.s.c. and $h$-continuous).

Definition 2.2. A multifunction $F: M \rightarrow \mathcal{B}(\mathbb{E})$ is called $h$-compact, if the set $\mathcal{R}_{\mathcal{B}(\mathbb{E})}(F)$ is precompact in $\mathcal{B}(\mathbb{E})$. If $\mathcal{R}_{\mathbb{E}}(F)$ is precompact in $\mathbb{E}$, then $F$ is called compact.

Remark 2.2. Since $\mathbb{E}$ and $\mathcal{B}(\mathbb{E})$ are complete metric spaces, a multifunction $F: M \rightarrow \mathcal{B}(\mathbb{E})$ is compact (resp. $h$-compact), if and only if $\overline{\mathcal{R}_{\mathbb{E}}(F)}$ is a compact subset of $\mathbb{E}\left(\right.$ resp. $\left.c l_{\mathcal{B}(\mathbb{E})} \mathcal{R}_{\mathcal{B}(\mathbb{E})}(F)\right)$ is a compact subset of $\mathcal{B}(\mathbb{E})$ ).

The following elementary proposition will be useful in the sequel.

Proposition 2.1. Let $F_{i}: M \rightarrow \mathcal{B}(\mathbb{E}), i=1,2$, be h-u.s.c. (resp. $h$ compact) and let $T$ be a non empty (resp. non empty compact) subset of $\mathbf{R}$. Then the multifunction $K: T \times T \times M \rightarrow \mathcal{B}(\mathbb{E})$ given by

$$
K\left(t_{1}, t_{2}, x\right)=\overline{t_{1} F_{1}(x)+t_{2} F_{2}(x)} \quad \text { for every } \quad\left(t_{1}, t_{2}, x\right) \in T \times T \times M
$$

is h-u.s.c. (resp. h-compact).

The class of $h$-compact multifunctions is strictly larger than the class of all compact multifunctions.

Proposition 2.2. For any $F: M \rightarrow \mathcal{B}(\mathbb{E})$ we have that $F$ is compact if and only if $F$ is h-compact and takes non empty compact values.

Proof. Set $A=\overline{\mathcal{R}_{\mathbb{E}}(F)}, \mathcal{A}=\operatorname{cl}_{\mathcal{B}(\mathbb{E})} \mathcal{R}_{\mathcal{B}(\mathbb{E})}(F)$. By Remark 2.2 it suffices to show that the following are equivalent:

(j) $A$ is a compact subset of $\mathbb{E}$,

(jj) $\mathcal{A}$ is a compact subset of $\mathcal{B}(\mathbb{E})$ and $F$ is $\mathcal{H}(\mathbb{E})$-valued.

Assume $(\mathrm{j}) . \quad F$ is $\mathcal{H}(\mathbb{E})$-valued, since $F(x) \subset A$ for every $x \in M$. Set $\mathcal{H}_{A}=\{Y \in \mathcal{H}(\mathbb{E}): Y \subset A\}$ and observe that $\mathcal{H}_{A}$, equipped with the metric $h$, 
is compact, because $A$ is so. Furthermore $\mathcal{H}(\mathbb{E})$ is closed in $\mathcal{B}(\mathbb{E})$, by Remark 2.1, and thus $\mathcal{A} \subset \mathcal{H}_{A}$. Consequently $\mathcal{A}$ is compact, proving (jj).

Assume (jj). For every $Y \in \mathcal{A}$ we have $Y \subset A$, since $A$ is closed in $\mathbb{E}$. Now let $\left\{y_{n}\right\} \subset A$. From the definition of $A$, there is a sequence $\left\{F\left(x_{n}\right)\right\} \subset \mathcal{A}$ such that

$$
d\left(y_{n}, F\left(x_{n}\right)\right) \rightarrow 0 \quad \text { as } n \rightarrow+\infty .
$$

Since $\mathcal{A}$ is compact, there exists a subsequence, say $\left\{F\left(x_{n}\right)\right\}$, and a set $Y \in \mathcal{A}$ such that

$$
h\left(F\left(x_{n}\right), Y\right) \rightarrow 0 \quad \text { as } n \rightarrow+\infty .
$$

From $d\left(y_{n}, Y\right) \leq d\left(y_{n}, F\left(x_{n}\right)\right)+h\left(F\left(x_{n}\right), Y\right)$, in view of (2.1) and (2.2), it follows that

$$
d\left(y_{n}, Y\right) \rightarrow 0 \quad \text { as } n \rightarrow+\infty .
$$

But $Y$ is compact in $\mathbb{E}$, and hence a subsequence, say $\left\{y_{n}\right\}$, converges to some $y \in Y$. As $Y \subset A$, it follows that $y \in A$. Thus $A$ is compact and (j) holds, completing the proof.

Let $\left\{\varphi_{n}\right\}$ and $\left\{\psi_{n}\right\}$ be sequences of multifunctions, where

$$
\varphi_{n}: M \rightarrow \mathcal{B}(\mathbb{E}) \text { and } \psi_{n}: T \times M \rightarrow \mathcal{B}(\mathbb{E}), T \text { a metric space. }
$$

Definition 2.3. A sequence $\left\{\varphi_{n}\right\}$ (resp. $\left\{\psi_{n}\right\}$ ) is said to be $e$-convergent to $F: M \rightarrow \mathcal{B}(\mathbb{E})$ if for every $x \in M$ and $\varepsilon>0$ there exist $\delta>0$ and $n_{0} \in \mathbf{N}$ such that

$$
\sup _{x^{\prime} \in U_{M}(x, \delta)} e\left(\varphi_{n}\left(x^{\prime}\right), F(x)\right)<\varepsilon, \quad\left(\text { resp. } \sup _{\left(t^{\prime}, x^{\prime}\right) \in T \times U_{M}(x, \delta)} e\left(\psi_{n}\left(t^{\prime}, x^{\prime}\right), F(x)\right)<\varepsilon\right)
$$

for every $n \geq n_{0}$.

In the above definition $\varphi_{n}$ and $\psi_{n}$ can be single valued. For brevity we write $\varphi_{n} \stackrel{e}{\longrightarrow} F$ to mean that $\left\{\varphi_{n}\right\}$ is $e$-convergent to $F$.

Definition 2.4. A sequence $\left\{f_{n}\right\}, \quad f_{n}: M \rightarrow \mathbb{E}$, is called collectively compact, if the set $\bigcup_{n \in \mathbf{N}} f_{n}(M)$ is a precompact subset of $\mathbb{E}$.

Given $F: M \rightarrow 2^{\mathbb{E}}$, a function $f: M \rightarrow \mathbb{E}$ satisfying $f(x) \in F(x)$ for every $x \in M$ is called a selection of $F$. The notion of a multivalued selection of $F$ is analogous. 


\section{§3. Topological Degree for Regular Multivalued Vector Fields}

In this section we define a topological degree for multivalued vector fields $I-F$, when $F$ is a $\mathcal{B}(\mathbb{E})$-valued regular multifunction (see Definition 3.2). Each $\mathcal{C}(\mathbb{E})$-valued $h$-u.s.c. and $h$-compact $F$, in particular each $\mathcal{K}(\mathbb{E})$-valued $h$-u.s.c. and compact $F$, is a regular multifunction. The topological degree we introduce reduces to that of Hukuhara [16], when $F$ is $h$-u.s.c. compact and takes its values in $\mathcal{K}(\mathbb{E})$.

Definition 3.1. $\quad$ Let $\varphi: M \rightarrow \mathcal{C}(\mathbb{E})$ be $h$-u.s.c. A sequence $\left\{f_{n}\right\}$ of continuous functions $f_{n}: M \rightarrow \mathbb{E}$ is called an admissible approximating sequence for $\varphi$ if (i) $\left\{f_{n}\right\}$ is collectively compact, and (ii) $\left\{f_{n}\right\}$ is $e$-convergent to $\varphi$. The family of all admissible approximating sequences for $\varphi$ will be denoted by $\mathcal{A}_{\varphi}$.

Proposition 3.1. $\quad$ Let $\varphi: M \rightarrow \mathcal{C}(\mathbb{E})$ be h-u.s.c. and h-compact. Then we have:

(i) $\varphi$ admits a h-u.s.c. and compact multivalued selection $\omega: M \rightarrow \mathcal{K}(\mathbb{E})$,

(ii) for every $x \in M, \varphi(x)=\bigcup\{\omega(x): \omega: M \rightarrow \mathcal{K}(\mathbb{E})$ is a h-u.s.c. compact selection of $\varphi\}$,

(iii) $\mathcal{A}_{\varphi} \neq \emptyset$.

Proof.

(i) Let $J: \mathcal{C}(\mathbb{E}) \rightarrow \mathcal{C}(\mathbb{E})$ be the multifunction given by

$$
J(X)=X \quad \text { for every } \quad X \in \mathcal{C}(\mathbb{E}) .
$$

Thus $J$ assigns to each $X$ in the metric space $(\mathcal{C}(\mathbb{E}), h)$ the non empty closed bounded and convex subset $X$ of $\mathbb{E} . J$ is $h$-continuous and hence lower semicontinuous in the sense of Michael [23], that is, for every open $V \subset \mathbb{E}$ the set $\{X \in \mathcal{C}(\mathbb{E}): J(X) \cap V \neq \emptyset\}$ is open in $\mathcal{C}(\mathbb{E})$. By the selection theorem of Michael [23] (see also Hu and Papageorgiou [15], p. 92) there is a continuous selection $s: \mathcal{C}(\mathbb{E}) \rightarrow \mathbb{E}$ such that

$$
s(X) \in X \quad \text { for every } X \in \mathcal{C}(\mathbb{E}) .
$$

Now consider the Filippov regularization of $s \circ \varphi$ that is the multifunction $\omega_{\text {so }}: M \rightarrow \mathcal{K}(\mathbb{E})$ defined by

$$
\omega_{s \circ \varphi}(x)=\bigcap_{n \in \mathbf{N}} \overline{c o}(s \circ \varphi)\left(U_{M}\left(x, \frac{1}{n}\right)\right) \quad \text { for every } x \in M .
$$


The set $(s \circ \varphi)(M)$ is precompact in $\mathbb{E}$, because the set $\mathcal{A}=c l_{\mathcal{C}(\mathbb{E})}\left(\mathcal{R}_{\mathcal{C}(\mathbb{E})}\right.$ $\times(\varphi))$ is compact in $\mathcal{C}(\mathbb{E})$ and $s$ is continuous. Clearly $\omega_{s \circ \varphi}(x) \in \mathcal{K}(\mathbb{E})$ for every $x \in M$.

The multifunction $\omega_{s \circ \varphi}$ is $h$-u.s.c. Let $x_{0} \in M$ and $\varepsilon>0$ be given. The sequence of compact sets $\overline{c o}(s \circ \varphi)\left(U_{M}\left(x_{0}, 1 / n\right)\right)$ is monotonic decreasing, by inclusion, and thus there exists $n_{0} \in \mathbf{N}$ such that $\overline{c o}(s \circ$ $\varphi)\left(U_{M}\left(x_{0}, 1 / n_{0}\right)\right) \subset \omega_{s \circ \varphi}\left(x_{0}\right)+\varepsilon U$. For every $x \in U_{M}\left(x_{0}, 1 / n_{0}\right)$ we have

$$
\begin{aligned}
\omega_{s \circ \varphi}(x) & =\bigcap_{n \in \mathbf{N}} \overline{c o}(s \circ \varphi)\left(U_{M}\left(x, \frac{1}{n}\right)\right) \\
& \subset \overline{c o}(s \circ \varphi)\left(U_{M}\left(x_{0}, \frac{1}{n_{0}}\right)\right) \subset \omega_{s \circ \varphi}\left(x_{0}\right)+\varepsilon U,
\end{aligned}
$$

and thus $\omega_{s \circ \varphi}$ is $h$-u.s.c. Further $\omega_{s \circ \varphi}$ is compact, because, for every $x \in M$, we have $\omega_{s \circ \varphi}(x) \subset \overline{c o}(\overline{(s \circ \varphi)(M)})$, where the latter set is compact, by Mazur's theorem.

It remains to show that $\omega_{s \circ \varphi}$ is a selection of $\varphi$. Let $x \in M$ and $\varepsilon>0$ be arbitrary. Fix $\delta>0$ so that $x^{\prime} \in U_{M}(x, \delta)$ implies $\varphi\left(x^{\prime}\right) \subset \varphi(x)+\varepsilon U$. For every $x^{\prime} \in U_{M}(x, \delta)$ we have $(s \circ \varphi)\left(x^{\prime}\right) \in \varphi\left(x^{\prime}\right) \subset \varphi(x)+(\varepsilon / 2) U$, thus for all $n$ large enough $\overline{c o}(s \circ \varphi)\left(U_{M}(x, 1 / n)\right) \subset \varphi(x)+\varepsilon U$ and so, a fortiori, $\omega s \circ \varphi(x) \subset \varphi(x)+\varepsilon U$. Therefore $\omega_{s \circ \varphi}(x) \subset \varphi(x)$. Letting $\omega=\omega_{s \circ \varphi}$, (i) is proved.

(ii) Let $x \in M$ and $y \in \varphi(x)$ be arbitrary, and let $\tilde{X}=\varphi(x)$. Let $\tilde{J}: \mathcal{C}(\mathbb{E}) \rightarrow$ $\mathcal{C}(\mathbb{E})$ be given by $\tilde{J}(X)=X$ if $X \neq \tilde{X}$ and $\tilde{J}(X)=\{y\}$ if $X=\tilde{X}$. $\tilde{J}$ is $h$-l.s.c., and hence also lower semicontinuous in the sense of Michael. Therefore there is a continuous function $s: \mathcal{C}(\mathbb{E}) \rightarrow \mathbb{E}$ such that $s(X) \in$ $\tilde{J}(X)$ for every $X \in \mathcal{C}(\mathbb{E})$. Since

$$
y=(s \circ \varphi)(x) \in \bigcap_{n \in \mathbf{N}} \overline{c o}(s \circ \varphi)\left(U_{M}\left(x, \frac{1}{n}\right)\right)=\omega_{s \circ \varphi}(x),
$$

it follows that

$$
\varphi(x) \subset\{\omega(x): \omega: M \rightarrow \mathcal{K}(\mathbb{E}) \text { is a h-u.s.c. compact selection of } \varphi\} .
$$

The opposite inclusion is obvious, and thus (ii) is proved.

(iii) Let $\omega: M \rightarrow \mathcal{K}(\mathbb{E})$ be a $h$-u.s.c. compact selection of $\varphi$. By the Hukuhara approximation theorem [16] (see also [5] and [15], p. 119) there is a sequence $\left\{\varphi_{n}\right\}$ of $h$-continuous multifunctions $\varphi_{n}: M \rightarrow \mathcal{K}(\mathbb{E})$ such that $(\mathrm{j}) \omega(x) \subset$ $\varphi_{n+1}(x) \subset \varphi_{n}(x)$ for every $x \in M$ and every $n \in \mathbf{N},(\mathrm{jj}) \mathcal{R}_{\mathbb{E}}\left(\varphi_{n}\right) \subset$ 
$\overline{c o} \mathcal{R}_{\mathbb{E}}(\omega)$ for every $n \in \mathbf{N}$, and (jjj) $h\left(\varphi_{n}(x), \omega(x)\right) \rightarrow 0$, as $n \rightarrow \infty$, for every $x \in M$.

For $n \in \mathbf{N}$, let $f_{n}: M \rightarrow \mathbb{E}$ be a continuous selection of $\varphi_{n}$. By $(\mathrm{jj}),\left\{f_{n}\right\}$ is collectively compact. Let $x \in M$ and $\varepsilon>0$ be arbitrary. By (jjj), there exists $n_{0} \in \mathbf{N}$ such that $n \geq n_{0}$ implies $\varphi_{n}(x) \subset \omega(x)+\varepsilon U$. Since $\varphi_{n_{0}}$ is $h$-continuous, there is $\delta>0$ such that $\varphi_{n_{0}}\left(x^{\prime}\right) \subset \varphi_{n_{0}}(x)+\varepsilon U \subset \omega(x)+2 \varepsilon U$ for every $x^{\prime} \in U_{M}(x, \delta)$. In view of $(\mathrm{j})$ we have

$$
f_{n}\left(x^{\prime}\right) \in \varphi_{n}\left(x^{\prime}\right) \subset \varphi_{n_{0}}\left(x^{\prime}\right) \subset \omega(x)+2 \varepsilon U \subset \varphi(x)+2 \varepsilon U
$$

for every $x^{\prime} \in U_{M}(x, \delta)$ and $n \geq n_{0}$, and thus $\left\{f_{n}\right\}$ is $e$-convergent to $\varphi$. Therefore $\left\{f_{n}\right\} \in \mathcal{A}_{\varphi}$, and (iii) is proved. This completes the proof.

Given $\varphi: A \rightarrow 2^{\mathbb{E}}$, where $\emptyset \neq A \subset \mathbb{E}$, and $r: \mathbb{E} \rightarrow \mathbb{E}$, we denote by $\overline{r \circ \varphi}$ the multifunction defined on $A$ with values in $2^{\mathbb{E}}$, defined by $\overline{(r \circ \varphi)}(x)=\overline{r(\varphi(x))}$, for every $x \in A$.

In the sequel $D$ will always denote a non empty open bounded subset of $\mathbb{E}, p$ a point of $\mathbb{E}$ and $I$ the identity map in $\mathbb{E}$. Further we set

$$
\begin{gathered}
\mathcal{L}_{\mathbb{E}}=\left\{r: \mathbb{E} \rightarrow \mathbb{E}: r \text { is Lipschitzean with constant } L_{r} \geq 0\right\}, \\
\mathcal{H}(\bar{D}, \mathcal{C}(\mathbb{E}))=\left\{\varphi: \bar{D} \rightarrow \mathcal{C}(\mathbb{E}): \varphi \text { is } h \text {-u.s.c. and } \mathcal{A}_{\varphi} \neq \emptyset\right\} .
\end{gathered}
$$

Remark 3.1. By Proposition 3.1, each $h$-u.s.c. and $h$-compact multifunction $\varphi: \bar{D} \rightarrow \mathcal{C}(\mathbb{E})$ is in $\mathcal{H}(\bar{D}, \mathcal{C}(\mathbb{E}))$.

Definition 3.2. $\quad$ A multifunction $F: \bar{D} \rightarrow \mathcal{B}(\mathbb{E})$ admits a regular representation if there exists a pair $(r, \varphi) \in \mathcal{L}_{\mathbb{E}} \times \mathcal{H}(\bar{D}, \mathcal{C}(\mathbb{E}))$ such that

$$
F(x)=\overline{(r \circ \varphi)}(x)
$$

for every $x \in \bar{D}$. In this case, $\overline{r \circ \varphi}: \bar{D} \rightarrow \mathcal{B}(\mathbb{E})$ is called a regular representation of $F$, and $F$ is called a regular multifunction. If $F$ is regular and admits a regular representation $\overline{r \circ \varphi}$, in which $r$ has inverse $r^{-1}$ Lipschitzean on $\mathbb{E}$, then $F$ is called a strongly regular multifunction.

It is evident that a regular multifunction is not necessarily convex valued.

For any regular multifunction $F: \bar{D} \rightarrow \mathcal{B}(\mathbb{E})$ we put

$\operatorname{Repr}(F)=\left\{(r, \varphi) \in \mathcal{L}_{\mathbb{E}} \times \mathcal{H}(\bar{D}, \mathcal{C}(\mathbb{E})): \overline{r \circ \varphi}\right.$ is a regular representation of $\left.F\right\}$

Remark 3.2. $\quad$ Each $h$-u.s.c. and h-compact multifunction $F: \bar{D} \rightarrow \mathcal{C}(\mathbb{E})$ is strongly regular and admits infinitely many regular representations $\overline{r_{\lambda} \circ \varphi_{\lambda}}$, where $r_{\lambda}=\lambda I$ and $\varphi_{\lambda}=\lambda^{-1} F, \lambda \neq 0$. 
Remark 3.3. Each regular multifunction $F: \bar{D} \rightarrow \mathcal{B}(\mathbb{E})$ is $h$-u.s.c.

We shall consider the following classes of multivalued vector fields:

$\mathcal{V}_{\text {comp }}(\bar{D}, \mathcal{K}(\mathbb{E}))=\{G: \bar{D} \rightarrow \mathcal{K}(\mathbb{E}): G=I-F$ and $F$ is $h$-u.s.c. and compact $\} ;$ $\mathcal{V}_{h-\operatorname{comp}}(\bar{D}, \mathcal{C}(\mathbb{E}))=\{G: \bar{D} \rightarrow \mathcal{C}(\mathbb{E}): G=I-F$ and $F$ is $h$-u.s.c. and $h$-compact $\} ;$ $\mathcal{V}_{r-\text { mult }}(\bar{D}, \mathcal{B}(\mathbb{E}))=\{G: \bar{D} \rightarrow \mathcal{B}(\mathbb{E}): G=I-F$ and $F$ is a regular multifunction $\}$.

Any $G$ which is in $\mathcal{V}_{\text {comp }}(\bar{D}, \mathcal{K}(\mathbb{E}))$, (resp. in $\mathcal{V}_{h-\text { comp }}(\bar{D}, \mathcal{C}(\mathbb{E})), \mathcal{V}_{r-\text { mult }}$ $\times(\bar{D}, \mathcal{B}(\mathbb{E})))$ is called a compact (resp. $h$-compact, regular) multivalued vector field.

Remark 3.4. We have

$$
\mathcal{V}_{\text {comp }}(\bar{D}, \mathcal{K}(\mathbb{E})) \subset \mathcal{V}_{h-\operatorname{comp}}(\bar{D}, \mathcal{C}(\mathbb{E})) \subset \mathcal{V}_{r-m u l t}(\bar{D}, \mathcal{B}(\mathbb{E}))
$$

where each inclusion is strict.

Proposition 3.2. Let $I-F \in \mathcal{V}_{r-m u l t}(\bar{D}, \mathcal{B}(\mathbb{E}))$ and let $\overline{r \circ \varphi}$ be a regular representation of $F$ for some $(r, \varphi) \in \operatorname{Repr}(F)$. We have

(i) For every $\left\{f_{n}\right\},\left\{\tilde{f}_{m}\right\} \in \mathcal{A}_{\varphi}$ the sequence $\left\{K_{n, m}\right\}$ of continuous functions $K_{n, m}:[0,1] \times \bar{D} \rightarrow \mathbb{E}$ given by

$$
K_{n, m}(t, x)=r\left((1-t) f_{n}(x)+t \tilde{f}_{m}(x)\right) \quad \text { for every }(t, x) \in[0,1] \times \bar{D}
$$

is collectively compact, and e-convergent to $F$ as $n, m \rightarrow+\infty$.

(ii) Let $\left\{f_{n}\right\},\left\{\tilde{f}_{n}\right\} \in \mathcal{A}_{\varphi}$. If for some point $p \in \mathbb{E}$ there exist sequences $\left\{f_{n_{k}}\right\}$, $\left\{\tilde{f}_{n_{k}}\right\}$, and $\left\{\left(t_{k}, x_{k}\right)\right\} \subset[0,1] \times \partial D$, such that

$$
p=x_{k}-r\left(\left(1-t_{k}\right) f_{n_{k}}\left(x_{k}\right)+t_{k} \tilde{f}_{n_{k}}\left(x_{k}\right)\right) \quad \text { for every } \quad k \in \mathbf{N},
$$

then there is a subsequence of $\left\{\left(t_{k}, x_{k}\right)\right\}$ which converges to a point $(t, x) \in$ $[0,1] \times \partial D$, where $x$ satisfies $p \in x-F(x)$.

Proof.

(i) $\left\{K_{n, m}\right\}$ is collectively compact, for $\left\{f_{n}\right\}$ and $\left\{\tilde{f}_{m}\right\}$ are so, and $r$ is continuous. To show that $\left\{K_{n, m}\right\}$ is $e$-convergent to $F$, take arbitrary $x \in \bar{D}$ and $\varepsilon>0$. Since $\left\{f_{n}\right\}$ and $\left\{\tilde{f}_{n}\right\}$ are $e$-convergent to $\varphi$, there exist $\delta>0$ and $n_{0} \in \mathbf{N}$ such that 


$$
f_{n}\left(x^{\prime}\right), \tilde{f}_{n}\left(x^{\prime}\right) \in \varphi(x)+\varepsilon U \quad \text { for every } \quad x^{\prime} \in U_{\bar{D}}(x, \delta), n \geq n_{0} .
$$

Hence, if $L_{r}$ is a Lipschitz constant for $r$, we have

$$
K_{n, m}\left(t, x^{\prime}\right) \in r(\varphi(x)+\varepsilon U) \subset F(x)+\varepsilon L_{r} U
$$

for every $\left(t, x^{\prime}\right) \in[0,1] \times U_{\bar{D}}(x, \delta)$ and $n, m \geq n_{0}$, and thus $\left\{K_{n, m}\right\}$ is $e$-convergent to $F$ as $n, m \rightarrow+\infty$.

(ii) Since $\left\{K_{n, m}\right\}$ is collectively compact, from (3.1) it follows that $\left\{\left(t_{k}, x_{k}\right)\right\}$ contains a subsequence, say $\left\{\left(t_{k}, x_{k}\right)\right\}$, which converges to a point $(t, x) \in$ $[0,1] \times \partial D$. Given $\varepsilon>0$, let $\delta>0$ and $n_{0} \in \mathbf{N}$ be such that (3.2) holds. Take $k_{0} \geq n_{0}$ so that $x_{k} \in U_{\bar{D}}(x, \delta)$ for all $k \geq k_{0}$. In view of (3.2), for every $k \geq k_{0}$ we have $f_{n_{k}}\left(x_{k}\right), \tilde{f}_{n_{k}}\left(x_{k}\right) \in \varphi(x)+\varepsilon U$, and hence

$$
r\left(\left(1-t_{k}\right) f_{n_{k}}\left(x_{k}\right)+t_{k} \tilde{f}_{n_{k}}\left(x_{k}\right)\right) \in r(\varphi(x)+\varepsilon U) \subset F(x)+\varepsilon L_{r} U .
$$

From (3.1) and (3.3) we have $p \in x_{k}-F(x)+\varepsilon L_{r} U$ for every $k \geq k_{0}$. Letting $k \rightarrow+\infty$ it follows that $p \in x-F(x)$, completing the proof.

Given a continuous and compact function $f: \bar{D} \rightarrow E$ and a point $p \notin$ $\cup_{x \in \partial D}(I-f)(x)$ we shall denote by $\operatorname{deg}(I-f, D, p)$ the Leray-Schauder topological degree of the vector field $I-f$ at $p$ relative to $D$ (see [20] and also [17], $[21],[26])$.

Proposition 3.3. Let $I-F \in \mathcal{V}_{r-m u l t}(\bar{D}, \mathcal{B}(\mathbb{E}))$ and let $p \notin \bigcup_{x \in \partial D}(I-$ $F)(x)$. Let $\overline{r \circ \varphi}$ be a regular representation of $F$ for some $(r, \varphi) \in \operatorname{Repr}(F)$. Then we have

(i) for every $\left\{f_{n}\right\} \in \mathcal{A}_{\varphi}$ there exists $n_{0} \in \mathbf{N}$ such that

$$
\operatorname{deg}\left(I-r \circ f_{n}, D, p\right)=\operatorname{deg}\left(I-r \circ f_{m}, D, p\right) \quad \text { for every } n, m \geq n_{0},
$$

(ii) for every $\left\{f_{n}\right\},\left\{\tilde{f}_{n}\right\} \in \mathcal{A}_{\varphi}$ there exists $n_{0} \in \mathbf{N}$ such that

$$
\operatorname{deg}\left(I-r \circ f_{n}, D, p\right)=\operatorname{deg}\left(I-r \circ \tilde{f}_{n}, D, p\right) \text { for every } n \geq n_{0} .
$$

Proof.

(i) For $n, m \in \mathbf{N}$, define $K_{n, m}:[0,1] \times \bar{D} \rightarrow \mathbb{E}$ by

$$
K_{n, m}(t, x)=r\left((1-t) f_{n}(x)+t f_{m}(x)\right) \quad \text { for every }(t, x) \in[0,1] \times \bar{D} .
$$

$K_{n, m}$ is continuous and satisfies 


$$
K_{n, m}(0, x)=\left(r \circ f_{n}\right)(x), \quad K_{n, m}(1, x)=\left(r \circ f_{m}\right)(x) \quad \text { for every } x \in \bar{D} .
$$

Further, by Proposition $3.2(\mathrm{i}),\left\{K_{n, m}\right\}$ is collectively compact and $e$ convergent to $F$ as $n, m \rightarrow+\infty$.

There is $n_{0} \in \mathbf{N}$ such that

$$
p \notin \bigcup_{n, m \geq n_{0}} \bigcup_{(t, x) \in[0,1] \times \partial D}\left(x-K_{n, m}(t, x)\right) .
$$

In the contrary case, there are sequences $\left\{f_{n_{k}}\right\},\left\{\tilde{f}_{m_{k}}\right\}$, and $\left\{\left(t_{k}, x_{k}\right)\right\} \subset$ $[0,1] \times \partial D$, such that

$$
p=x_{k}-r\left(\left(1-t_{k}\right) f_{n_{k}}\left(x_{k}\right)+t_{k} f_{m_{k}}\left(x_{k}\right)\right) \quad \text { for every } k \in \mathbf{N} .
$$

Proposition 3.2 (ii) implies that a subsequence of $\left\{\left(t_{k}, x_{k}\right)\right\}$, say $\left\{\left(t_{k}, x_{k}\right)\right\}$, converges to a point $(t, x) \in[0,1] \times \partial D$, where $x$ satisfies $p \in x-F(x)$, a contradiction. Hence, for some $n_{0} \in \mathbf{N},(3.7)$ holds.

Now, for each $n, m \geq n_{0}, K_{n, m}$ is continuous compact and satisfies (3.7). By the homotopy property of the Leray-Schauder topological degree, in view of (3.6), (3.4) follows, and so (i) is proved.

(ii) The proof of (3.5) is similar, if one defines $K_{n}:[0,1] \times \bar{D} \rightarrow \mathbb{E}$ by

$$
K_{n}(t, x)=r\left((1-t) f_{n}(x)+t \tilde{f}_{n}(x)\right) \text { for every }(t, x) \in[0,1] \times \bar{D} .
$$

This completes the proof.

Definition 3.3. Let $I-F \in \mathcal{V}_{r-m u l t}(\bar{D}, \mathcal{B}(\mathbb{E}))$ and let $p \notin \bigcup_{x \in \partial D}(I-$ $F)(x)$. Let $\overline{r \circ \varphi}$ be a regular representation of $F$ for some $(r, \varphi) \in \operatorname{Repr}(F)$. For arbitrary $\left\{f_{n}\right\} \in \mathcal{A}_{\varphi}$ the topological degree $d(I-\overline{r \circ \varphi}, D, p)$ of $I-\overline{r \circ \varphi}$ at $p$ relative to $D$ is defined by

$$
d(I-\overline{r \circ \varphi}, D, p)=\lim _{n \rightarrow \infty} \operatorname{deg}\left(I-r \circ f_{n}, D, p\right) .
$$

The topological degree $\operatorname{Deg}(I-F, D, p)$ of $I-F$ at $p$ relative to $D$ is defined by

$$
\operatorname{Deg}(I-F, D, p)=\{d(I-\overline{r \circ \varphi}, D, p):(r, \varphi) \in \operatorname{Repr}(F)\} .
$$

Remark 3.5. The limit (3.8) exists and is finite by Proposition 3.3 (i), and it is independent of $\left\{f_{n}\right\} \in \mathcal{A}_{\varphi}$ by Proposition 3.3 (ii).

Proposition 3.4. Let $I-F \in \mathcal{V}_{r-m u l t}(\bar{D}, \mathcal{B}(\mathbb{E}))$ and let $p \notin \bigcup_{x \in \partial D}(I-$ $F)(x)$. If, in addition, $F$ is a strongly regular multifunction, then $\operatorname{Deg}(I-$ $F, D, p)$ is singleton. 
Proof. Let $\overline{r \circ \varphi}$ be an arbitrary regular representation of $F$ for some $(r, \varphi) \in \operatorname{Repr}(F)$. By hypothesis, there exists a regular representation $\overline{\tilde{r} \circ \tilde{\varphi}}$ of $F$ for some $(\tilde{r}, \tilde{\varphi}) \in \operatorname{Repr}(F)$, where $\tilde{r}$ has inverse $\tilde{r}^{-1}$, Lipschitzean on $\mathbb{E}$. Let $\left\{f_{n}\right\} \in \mathcal{A}_{\varphi}$ and $\left\{\tilde{f}_{n}\right\} \in \mathcal{A}_{\tilde{\varphi}}$ be arbitrary. For $n \in \mathbf{N}$ define $g_{n}: \bar{D} \rightarrow \mathbb{E}$ by $g_{n}(x)=\left(\tilde{r}^{-1} \circ r \circ f_{n}\right)(x), x \in \bar{D}$.

We have $\left\{g_{n}\right\} \in \mathcal{A}_{\tilde{\varphi}}$. Clearly, $g_{n}$ is continuous, and the sequence $\left\{g_{n}\right\}$ is collectively compact, for $\left\{f_{n}\right\}$ is so and $\tilde{r}^{-1} \circ r$ is continuous. Moreover, $\left\{g_{n}\right\}$ is $e$-convergent to $\tilde{\varphi}$. Let $x \in \bar{D}$ and $\varepsilon>0$ be given. Since $f_{n} \stackrel{e}{\longrightarrow} \varphi$, there are $\delta>0$ and $n_{0} \in \mathbf{N}$ such that

$$
f_{n}\left(x^{\prime}\right) \in \varphi(x)+\varepsilon U \text { for every } x^{\prime} \in U_{\bar{D}}(x, \delta), n \geq n_{0} .
$$

Let $L_{r}$ and $L_{\tilde{r}^{-1}}$ be Lipschitz constants for $r$ and $\tilde{r}^{-1}$, respectively. In view of (3.9), for every $x^{\prime} \in U_{\bar{D}}(x, \delta)$ and $n \geq n_{0}$ we have

$$
g_{n}\left(x^{\prime}\right) \in \tilde{r}^{-1}(r(\varphi(x)+\varepsilon U)) \subset \tilde{r}^{-1}\left(F(x)+\varepsilon L_{r} U\right) \subset \tilde{\varphi}(x)+\varepsilon L_{r} L_{\tilde{r}^{-1}} U,
$$

and so $\left\{g_{n}\right\}$ is $e$-convergent to $\tilde{\varphi}$. Hence $\left\{g_{n}\right\} \in \mathcal{A}_{\tilde{\varphi}}$.

For $n \in \mathbf{N}$ define $K_{n}:[0,1] \times \bar{D} \rightarrow \mathbb{E}$ by

$$
K_{n}(t, x)=\tilde{r}\left((1-t) g_{n}(x)+t \tilde{f}_{n}(x)\right) \quad \text { for every }(t, x) \in[0,1] \times \bar{D} .
$$

Since $\left\{g_{n}\right\},\left\{\tilde{f}_{n}\right\} \in \mathcal{A}_{\tilde{\varphi}}$, by Proposition $3.2(\mathrm{i})(n=m)$, the sequence $\left\{K_{n}\right\}$ is collectively compact and $e$-convergent to $F$. Further, by virtue of Proposition 3.2 (ii), one can show that there exists $n_{0} \in \mathbf{N}$ such that

$$
p \notin \bigcup_{n \geq n_{0}} \bigcup_{(t, x) \in[0,1] \times \bar{D}}\left(x-K_{n}(t, x)\right) .
$$

The $K_{n}$ are continuous compact and satisfy (3.10) and (3.11), and thus by the homotopy property of the Leray-Schauder topological degree we have

$$
\operatorname{deg}\left(I-r \circ f_{n}, D, p\right)=\operatorname{deg}\left(I-\tilde{r} \circ \tilde{f}_{n}, D, p\right) \text { for every } n \geq n_{0} .
$$

But $\left\{f_{n}\right\} \in \mathcal{A}_{\varphi}$ and $\left\{\tilde{f}_{n}\right\} \in \mathcal{A}_{\tilde{\varphi}}$, and hence by Definition 3.3 and Remark 3.5 it follows

$$
d(I-\overline{r \circ \varphi}, D, p)=d(I-\overline{\tilde{r} \circ \tilde{\varphi}}, D, p) .
$$

As $(r, \varphi) \in \operatorname{Repr}(F)$ is arbitrary, the topological degree $\operatorname{Deg}(I-F, D, p)$ is singleton. This completes the proof.

Remark 3.6. Since each $h$-u.s.c. and $h$-compact multifunction $F: \bar{D} \rightarrow$ $\mathcal{C}(\mathbb{E})$ is strongly regular, the conclusion of Proposition 3.4 remains valid if $I-F \in \mathcal{V}_{h-\operatorname{comp}}(\bar{D}, \mathcal{C}(\mathbb{E}))$ and, in particular, if $I-F \in \mathcal{V}_{\text {comp }}(\bar{D}, \mathcal{K}(\mathbb{E}))$. 
Proposition 3.5. Let $I-F \in \mathcal{V}_{\text {comp }}(\bar{D}, \mathcal{K}(\mathbb{E}))$ and let $p \notin \bigcup_{x \in \partial D}(I-$ $F)(x)$. Then $\operatorname{Deg}(I-F, D, p)$ coincides with the Hukuhara topological degree of $I-F$ at $p$ relative to $D$.

Proof. Clearly $\operatorname{Deg}(I-F, D, p)$ is singleton, by Remark 3.6, and $(I, F) \in$ $\operatorname{Repr}(F)$, by Remark 3.2. Let $\left\{f_{n}\right\}$ be constructed as in the proof of Proposition 3.1 (iii) (with $\omega=\varphi=F$ ), and thus $\left\{f_{n}\right\} \in \mathcal{A}_{F}$. By Definition 3.3 we have

$$
\operatorname{Deg}(I-F, D, p)=d(I-F, D, p)=\lim _{n \rightarrow \infty} \operatorname{deg}\left(I-f_{n}, D, p\right),
$$

from which the result follows, as the limit is the Hukuhara topological degree of $I-F$ at $p$ relative to $D$.

Remark 3.7. If $F: \bar{D} \rightarrow E$ is continuous and compact, the Hukuhara topological degree reduces to that of Leray and Schauder.

\section{$\S 4$. Properties of the Topological Degree}

In this section we establish a few properties of the topological degree $\operatorname{Deg}(I-F, D, p)$ that are useful in fixed point theory.

Proposition 4.1 (invariance under homotopy). Let $I-F_{1}, I-F_{2} \in$ $\mathcal{V}_{r-\text { mult }}(\bar{D}, \mathcal{B}(\mathbb{E}))$ and suppose that the multifunction $H:[0,1] \times \bar{D} \rightarrow \mathcal{B}(\mathbb{E})$ given by

$$
H(t, x)=\overline{(1-t) F_{1}(x)+t F_{2}(x)} \quad \text { for every }(t, x) \in[0,1] \times \bar{D}
$$

satisfies $p \notin \bigcup_{(t, x) \in[0,1] \times \partial D}(x-H(t, x))$. Then we have

$$
\operatorname{Deg}\left(I-F_{1}, D, p\right)=\operatorname{Deg}\left(I-F_{2}, D, p\right),
$$

where both sides are singletons.

Proof. For $i=1,2$, let $\overline{r_{i} \circ \varphi_{i}}$ be a regular representation of $F_{i}$ for some $\left(r_{i}, \varphi_{i}\right) \in \operatorname{Repr}\left(F_{i}\right)$. Let $\left\{f_{n}^{i}\right\} \in \mathcal{A}_{\varphi_{i}}, i=1,2$. For $n \in \mathbf{N}$ define $K_{n}:[0,1] \times$ $\bar{D} \rightarrow \mathbb{E}$ by

$$
K_{n}(t, x)=(1-t)\left(r_{1} \circ f_{n}^{1}\right)(x)+t\left(r_{2} \circ f_{n}^{2}\right)(x) \text { for every }(t, x) \in[0,1] \times \bar{D} .
$$

Each $K_{n}$ is continuous and the sequence $\left\{K_{n}\right\}$ is collectively compact, for $\left\{f_{n}^{1}\right\}$ and $\left\{f_{n}^{2}\right\}$ are so. There is $n_{0} \in \mathbf{N}$ such that

$$
p \notin \bigcup_{n \geq n_{0}} \bigcup_{(t, x) \in[0,1] \times \partial D}\left(x-K_{n}(t, x)\right) .
$$


In the contrary case there are sequences $\left\{f_{n_{k}}^{1}\right\},\left\{f_{n_{k}}^{2}\right\}$, and $\left\{\left(t_{k}, x_{k}\right)\right\} \subset[0,1] \times$ $\partial D$, such that

$$
p=x_{k}-\left(\left(1-t_{k}\right)\left(r_{1} \circ f_{n_{k}}^{1}\right)\left(x_{k}\right)+t_{k}\left(r_{2} \circ f_{n_{k}}^{2}\right)\left(x_{k}\right)\right) \quad \text { for every } \quad k \in \mathbf{N} .
$$

Since $\left\{K_{n}\right\}$ is collectively compact, $\left\{\left(t_{k}, x_{k}\right)\right\}$ contains a subsequence, say $\left\{\left(t_{k}, x_{k}\right)\right\}$, which converges to a point $(t, x) \in[0,1] \times \partial D$. It will be shown that

$$
p \in x-H(t, x) .
$$

Let $\varepsilon>0$. For $i=1,2,\left\{f_{n}^{i}\right\}$ is $e$-convergent to $\varphi_{i}$, and thus there are $\delta>0$ and $n_{0} \in \mathbf{N}$ such that

$$
f_{n}^{1}\left(x^{\prime}\right) \in \varphi_{1}(x)+\varepsilon U, \quad f_{n}^{2}\left(x^{\prime}\right) \in \varphi_{2}(x)+\varepsilon U \text { for every } x^{\prime} \in U_{\bar{D}}(x, \delta), n \geq n_{0} .
$$

Now fix $k_{0} \geq n_{0}$ so that $x_{k} \in U_{\bar{D}}(x, \delta)$ for all $k \geq k_{0}$. Hence

$$
f_{n_{k}}^{1}\left(x_{k}\right) \in \varphi_{1}(x)+\varepsilon U, \quad f_{n_{k}}^{2}\left(x_{k}\right) \in \varphi_{2}(x)+\varepsilon U \text { for every } k \geq k_{0} .
$$

From (4.2), in view of (4.4), for every $k \geq k_{0}$ we have

$$
\begin{aligned}
p & \left.\in x_{k}-\left(\left(1-t_{k}\right) r_{1}\left(\varphi_{1}(x)+\varepsilon U\right)\right)+t_{k} r_{2}\left(\varphi_{2}(x)+\varepsilon U\right)\right) \\
& \subset x_{k}-\left(\left(1-t_{k}\right)\left(F_{1}(x)+\varepsilon L_{r_{1}} U\right)+t_{k}\left(F_{2}(x)+\varepsilon L_{r_{2}} U\right)\right) \\
& \subset x_{k}-H\left(t_{k}, x\right)+\varepsilon\left(L_{r_{1}}+L_{r_{2}}\right) U,
\end{aligned}
$$

where $L_{r_{i}}$ is a Lipschitz constant for $r_{i}, i=1,2$. Letting $k \rightarrow+\infty,(4.3)$ follows, a contradiction. Thus, for some $n_{0} \in \mathbf{N},(4.1)$ holds.

The $K_{n}$ are continuous compact and satisfy (4.1), and thus by the homotopy property of the Leray-Schauder topological degree we have

$$
\operatorname{deg}\left(I-r_{1} \circ f_{n}^{1}, D, p\right)=\operatorname{deg}\left(I-r_{2} \circ f_{n}^{2}, D, p\right) \quad \text { for every } n \geq n_{0} .
$$

As $\left\{f_{n}^{1}\right\} \in \mathcal{A}_{\varphi_{1}}$ and $\left\{f_{n}^{2}\right\} \in \mathcal{A}_{\varphi_{2}}$, letting $n \rightarrow+\infty$ it follows that $d(I-$ $\left.\overline{r_{1} \circ \varphi_{1}}, D, p\right)=d\left(I-\overline{r_{2} \circ \varphi_{2}}, D, p\right)$. But $\left(r_{1}, \varphi_{1}\right) \in \operatorname{Repr}\left(F_{1}\right)$ and $\left(r_{2}, \varphi_{1}\right) \in$ $\operatorname{Repr}\left(F_{2}\right)$ are arbitrary, and hence

$$
\operatorname{Deg}\left(I-F_{1}, D, p\right)=\operatorname{Deg}\left(I-F_{2}, D, p\right),
$$

where, clearly, both sides are singletons. This completes the proof.

Proposition 4.2 (inclusion solving property). Let $I-F \in \mathcal{V}_{r-\text { mult }}(\bar{D}$, $\mathcal{B}(\mathbb{E}))$, let $p \notin \bigcup_{x \in \partial D}(I-F)(x)$, and suppose that $\operatorname{Deg}(I-F, D, p) \neq\{0\}$. Then there exists an $x \in D$ such that

$$
p \in x-F(x) .
$$


Proof. Since $\operatorname{Deg}(I-F, D, p) \neq\{0\}$, there is a regular representation $\overline{r \circ \varphi}$ of $F$ for some $(r, \varphi) \in \operatorname{Repr}(F)$, such that $d(I-\overline{r \circ \varphi}, D, p) \neq 0$. Let $\left\{f_{n}\right\} \in \mathcal{A}_{\varphi}$. By Definition 3.3 and Proposition 3.3 (i), there exists $m_{0} \in \mathbf{N}$ such that $\operatorname{deg}\left(I-r \circ f_{n}, D, p\right) \neq 0$ for every $n \geq m_{0}$. By a property of the Leray-Schauder topological degree, for every $n \geq m_{0}$ there exists $x_{n} \in D$ such that

$$
p=x_{n}-\left(r \circ f_{n}\right)\left(x_{n}\right) .
$$

As $\left\{f_{n}\right\}$ is collectively compact, $\left\{x_{n}\right\}$ contains a subsequence, say $\left\{x_{n}\right\}$, which converges to a point $x \in \bar{D}$. Let $\varepsilon>0$ be arbitrary. Since $\left\{f_{n}\right\}$ is $e$-convergent to $\varphi$, there are $\delta>0$ and $m_{1} \geq m_{0}$ such that

$$
f_{n}\left(x^{\prime}\right) \in \varphi(x)+\varepsilon U \text { for every } x^{\prime} \in U_{\bar{D}}(x, \delta), n \geq m_{1} .
$$

Now take $n_{0} \geq m_{1}$ so that $x_{n} \in U_{\bar{D}}(x, \delta)$ for all $n \geq n_{0}$. From (4.6), in view of (4.7), for every $n \geq n_{0}$ we have

$$
p=x_{n}-r\left(f_{n}\left(x_{n}\right)\right) \in x_{n}-r(\varphi(x)+\varepsilon U) \subset x_{n}-F(x)+\varepsilon L_{r} U,
$$

where $L_{r}$ is a Lipschitz constant for $r$. Letting $n \rightarrow+\infty$, (4.5) follows and, clearly, $x \in D$. This completes the proof.

Proposition 4.3 (normalization). If $p \in D$ then $\operatorname{Deg}(I, D, p)=1$.

Proof. The Hukuhara topological degree has this property, and thus the statement follows from Proposition 3.5.

Proposition 4.4 (continuity in $p$ ). $\quad$ Let $I-F \in \mathcal{V}_{r-m u l t}(\bar{D}, \mathcal{B}(\mathbb{E}))$ and let $p, q \in A$, where $A$ is an open component of $\mathbb{E} \backslash \cup_{x \in \partial D}(I-F)(x)$. Then we have

$$
\operatorname{Deg}(I-F, D, p)=\operatorname{Deg}(I-F, D, q)
$$

Proof. Let $(r, \varphi) \in \operatorname{Repr}(F)$ be arbitrary, and let $\left\{f_{n}\right\} \in \mathcal{A}_{\varphi}$. Let $\gamma$ : $[0,1] \rightarrow \mathbb{E}$ be a continuous path contained in $A$, joining $p$ and $q$. For $\varepsilon>0$ put $C_{\varepsilon}=\bigcup_{t \in[0,1]} U(\gamma(t), \varepsilon) . C_{\varepsilon}$ is open and connected, and $C_{\varepsilon} \subset A$, provided that $\varepsilon$ is small enough.

There exist $\varepsilon>0$ and $n_{0} \in \mathbf{N}$ such that

$$
C_{\varepsilon} \cap\left(\bigcup_{n \geq n_{0}} \bigcup_{x \in \partial D}\left(I-r \circ f_{n}\right)(x)\right)=\emptyset .
$$


In the contrary case, there exist sequences $\left\{f_{n_{k}}\right\}$, and $\left\{\left(t_{k}, x_{k}\right)\right\} \subset[0,1] \times \partial D$, such that

$$
\gamma\left(t_{k}\right) \in x_{k}-\left(r \circ f_{n_{k}}\right)\left(x_{k}\right)+\frac{1}{k} U \quad \text { for every } k \in \mathbf{N} .
$$

As $\left\{f_{n}\right\}$ is collectively compact and $\gamma([0,1])$ is compact, there exist subsequences, say $\left\{x_{k}\right\}$ and $\left\{\gamma\left(t_{k}\right)\right\}$, converging respectively to $x \in \partial D$ and $y \in$ $\gamma([0,1])$. But $\left\{f_{n}\right\}$ is $e$-convergent to $\varphi$, and thus given $\varepsilon>0$ there exist $\delta>0$ and $m_{0} \in \mathbf{N}$ such that $f_{n}\left(x^{\prime}\right) \in \varphi(x)+\varepsilon U$ for every $x^{\prime} \in U_{\bar{D}}(x, \delta)$ and $n \geq m_{0}$. Fix a $k_{0} \geq m_{0}$ so that $x_{k} \in U_{\bar{D}}(x, \delta)$ for all $k \geq k_{0}$. Hence

$$
f_{n_{k}}\left(x_{k}\right) \in \varphi(x)+\varepsilon U \quad \text { for every } k \geq k_{0} .
$$

From (4.10) and (4.11) we obtain

$\gamma\left(t_{k}\right) \in x_{k}-r(\varphi(x)+\varepsilon U)+\frac{1}{k} U \subset x_{k}-F(x)+\left(\varepsilon L_{r}+\frac{1}{k}\right) U$ for every $k \geq k_{0}$,

where $L_{r}$ is a Lipschitz constant for $r$. Letting $k \rightarrow+\infty$ it follows that $y \in$ $x-F(x)$, a contradiction, as $x \in \partial D$ and $y \in A$. Therefore for some $\varepsilon>0$ and $n_{0} \in \mathbf{N},(4.9)$ holds.

Now (4.9) implies

$$
C_{\varepsilon} \subset \mathbb{E} \backslash \bigcup_{x \in \partial D}\left(I-r \circ f_{n}\right)(x) \text { for every } n \geq n_{0} .
$$

Since $p$ and $q$ are in $C_{\varepsilon}$, by a property of the Leray-Schauder topological degree one has $\operatorname{deg}\left(I-r \circ f_{n}, D, p\right)=\operatorname{deg}\left(I-r \circ f_{n}, D, q\right)$ for every $n \geq n_{0}$. Hence, by Definition 3.3, $d(I-\overline{r \circ \varphi}, D, p)=d(I-\overline{r \circ \varphi}, D, q)$. As $(r, \varphi) \in \operatorname{Repr}(F)$ is arbitrary, (4.8) follows, completing the proof.

\section{§5. Applications to Fixed Point Theory}

In this section we use our topological degree to obtain simple proofs of fixed point theorems for regular multifunctions. A result of Borsuk's type for $\mathcal{C}(\mathbb{E})$-valued multifunctions will be considered as well.

For any $F: A \rightarrow 2^{\mathbb{E}}$, where $\emptyset \neq A \subset \mathbb{E}$, a point $x \in A$ such that $x \in F(x)$ is called a fixed point of $F$. In the sequel 0 denotes the zero of the space $\mathbb{E}$.

Proposition 5.1. $\quad$ Let $C$ be a convex open bounded subset of $\mathbb{E}$ and let $0 \in C$. Let $F: \bar{C} \rightarrow \mathcal{B}(\mathbb{E})$ be a regular multifunction satisfying $F(x) \subset \bar{C}$ for every $x \in \bar{C}$. Then $F$ has a fixed point. 
Proof. Without loss of generality we suppose that

$$
0 \notin \bigcup_{x \in \partial C}(I-F)(x) \text {. }
$$

Now define $H:[0,1] \times \bar{C} \rightarrow \mathcal{B}(\mathbb{E})$ by $H(t, x)=t F(x)$ for every $(t, x) \in[0,1] \times \bar{C}$, and observe that

$$
0 \notin \bigcup_{(t, x) \in[0,1] \times \partial C}(x-H(t, x)) .
$$

In fact, if (5.2) fails, for some $x \in \partial C$ and $t \in(0,1)$ we have $x \in t F(x)$, which is impossible because $F(x) \subset \bar{C}$ and 0 is an interior point of $\bar{C}$. Clearly $I$ and $I-F$ are both in $\mathcal{V}_{r-\text { mult }}(\bar{C}, \mathcal{B}(\mathbb{E}))$. By Proposition 4.1, taking into account (5.2) and Proposition 4.3, one has $\operatorname{Deg}(I-F, C, 0)=1$. Hence, by Proposition $4.2, F$ has a fixed point, completing the proof.

Proposition 5.2. Let $C$ be as in Proposition 5.1. Let $F: \bar{C} \rightarrow \mathcal{B}(\mathbb{E})$ be a regular multifunction which satisfies the condition

$$
\inf _{y \in F(x)}\left[\|y-x\|^{2}-\|y\|^{2}+\|x\|^{2}\right] \geq 0 \quad \text { for every } x \in \partial C .
$$

Then $F$ has a fixed point.

Proof. Without loss of generality we suppose that (5.1) is satisfied. With $H$ as in the proof of Proposition 5.1, (5.2) holds. In the contrary case, for some $x \in \partial C$ and $t \in(0,1)$ we have $x \in t F(x)$. Now (5.3) implies

$$
\left\|\frac{x}{t}-x\right\|^{2} \geq\left\|\frac{x}{t}\right\|^{2}-\|x\|^{2}
$$

and therefore $(1 / t-1)^{2} \geq 1 / t^{2}-1$, for $x \neq 0$. As $t \in(0,1)$, a contradiction follows, and thus (5.2) holds. The conclusion is as for Proposition 5.1.

Proposition 5.3. Let $C$ be as in Proposition 5.1. Let $F: \bar{C} \rightarrow \mathcal{B}(\mathbb{E})$ be a regular multifunction which satisfies the Leray-Schauder condition

$$
x \in t F(x) \quad \text { for some } \quad x \in \partial C \text { and } t>0 \text { implies } t=1 .
$$

Then $F$ has a fixed point.

Proof. Without loss of generality we suppose that (5.1) is satisfied. With $H$ as in the proof of Proposition 5.1, (5.2) holds. Otherwise, for some $x \in \partial C$ and $t \in(0,1]$ we have $x \in t F(x)$ and, by (5.4), $x \in F(x)$, a contradiction to (5.1). The conclusion follows as for Proposition 5.1. 
Proposition 5.4. $\quad$ Let $C$ be a non empty open bounded subset of $\mathbb{E}$. Let $F: \bar{C} \rightarrow \mathcal{B}(\mathbb{E})$ be a regular multifunction which satisfies the Rothe condition:

$$
\begin{gathered}
\text { there exists } u \in C \text { such that } t(x-u) \notin F(x)-u \\
\text { for every } x \in \partial C \text { and } t>1 .
\end{gathered}
$$

Then $F$ has a fixed point.

Proof. Without loss of generality we suppose that (5.1) holds. Clearly $I-u$ and $I-F$ are in $\mathcal{V}_{r-a p p r}(\bar{C}, \mathcal{B}(\mathbb{E}))$. Let $H:[0,1] \times \bar{C} \rightarrow \mathcal{B}(\mathbb{E})$ be given by $H(t, x)=(1-t) u+t F(x)$ for every $(t, x) \in[0,1] \times \bar{C}$. $H$ satisfies (5.2). In the contrary case, for some $x \in \partial C$ and $t \in[0,1]$ we have $x \in(1-t) u+t F(x)$, and so $x-u \in t(F(x)-u)$. But $t \in(0,1)$, as $u \notin \partial C$ and $x \notin F(x)$, whence $(1 / t)(x-u) \in F(x)-u$, a contradiction to (5.5). Therefore (5.2) holds. By Proposition 4.1, in view of (5.2) and Proposition 3.5, we have $\operatorname{Deg}(I-F, C, 0)=$ $\operatorname{Deg}(I-u, C, 0)=1$. By Proposition $4.3, F$ has a fixed point, completing the proof.

The fixed point results of Propositions 5.1-5.4 are variants of theorems established by Kakutani [18] and Ky Fan [7], Altman [1], Leray and Schauder [20] and Rothe [25].

We conclude with a Borsuk's type result for $\mathcal{C}(\mathbb{E})$-valued multifunctions. Under different assumptions, multivalued versions of Borsuk's theorems have been previously obtained by Granas [14], Ma [22] and Lasry and Robert [19].

Proposition 5.5. $\quad$ Let $C$ be an open bounded symmetric subset of $\mathbb{E}$, and let $0 \in C$. Let $F: \bar{C} \rightarrow \mathcal{C}(\mathbb{E})$ be a h-u.s.c. and h-compact multifunction satisfying $0 \notin \bigcup_{x \in \partial C}(I-F)(x)$. If, in addition, $F$ is odd on $\partial C$, i.e. $F(x)=$ $-F(-x)$ for every $x \in \partial C$, then

$$
\operatorname{Deg}(I-F, C, 0)=1 \quad(\bmod 2) .
$$

Moreover, $F$ has a fixed point.

Proof. By Proposition 3.1, $\mathcal{A}_{F}$ is non empty. Let $\left\{f_{n}\right\} \in \mathcal{A}_{F}$. For $n \in \mathbf{N}$ define $g_{n}: \bar{C} \rightarrow \mathbb{E}$ and $K_{n}:[0,1] \times \bar{C} \rightarrow \mathbb{E}$ by

$$
g_{n}(x)=\frac{f_{n}(x)-f_{n}(-x)}{2} \quad \text { for every } \quad x \in \bar{C},
$$

and

$$
K_{n}(t, x)=(1-t) f_{n}(x)+\operatorname{tg}_{n}(x) \quad \text { for every } \quad(t, x) \in[0,1] \times \bar{C}
$$


Clearly $g_{n}$ is odd on $\bar{C}$.

There is $n_{0} \in \mathbf{N}$ such that

$$
0 \notin \bigcup_{n \geq n_{0}} \bigcup_{(t, x) \in[0,1] \times \partial C}\left(x-K_{n}(t, x)\right) .
$$

In the contrary case there are sequences $\left\{f_{n_{k}}\right\}$, and $\left\{\left(t_{k}, x_{k}\right)\right\} \subset[0,1] \times \partial C$, such that

$$
x_{k}=\left(1-t_{k}\right) f_{n_{k}}\left(x_{k}\right)+t_{k} \frac{f_{n_{k}}\left(x_{k}\right)-f_{n_{k}}\left(-x_{k}\right)}{2} \text { for every } \quad k \in \mathbf{N} .
$$

Since $\left\{f_{n}\right\}$ is collectively compact, $\left\{\left(t_{k}, x_{k}\right)\right\}$ contains a subsequence, say $\left\{\left(t_{k}\right.\right.$, $\left.\left.x_{k}\right)\right\}$, which converges to a point $(t, x) \in[0,1] \times \partial C$.

On the other hand $\left\{f_{n}\right\}$ is $e$-convergent to $F$ and thus, given $\varepsilon>0$, there exist $\delta>0$ and $n_{0} \in \mathbf{N}$ such that

$$
\begin{array}{cll}
f_{n}\left(x^{\prime}\right) \in F(x)+\varepsilon U & \text { for every } & x^{\prime} \in U_{\bar{C}}(x, \delta), n \geq n_{0}, \\
f_{n}\left(x^{\prime}\right) \in F(-x)+\varepsilon U & \text { for every } & x^{\prime} \in U_{\bar{C}}(-x, \delta), n \geq n_{0} .
\end{array}
$$

Fix $k_{0} \geq n_{0}$ so that $x_{k} \in U_{\bar{C}}(x, \delta)$ and $-x_{k} \in U_{\bar{C}}(-x, \delta)$ for all $k \geq k_{0}$. Hence

$$
f_{n_{k}}\left(x_{k}\right) \in F(x)+\varepsilon U \quad f_{n_{k}}\left(-x_{k}\right) \in F(-x)+\varepsilon U \quad \text { for every } \quad k \geq k_{0} .
$$

From (5.8), in view of (5.9) and the assumption that $F$ is odd on $\partial C$, one has

$$
x_{k} \in\left(1-t_{k}\right)(F(x)+\varepsilon U)+t_{k} \frac{(F(x)+\varepsilon U)-(F(-x)+\varepsilon U)}{2}=F(x)+\varepsilon U .
$$

Letting $k \rightarrow+\infty$, it follows that $x \in F(x)$, a contradiction. Therefore, for some $n_{0} \in \mathbf{N},(5.7)$ holds.

In view of (5.7), the homotopy property of the Leray-Schauder topological degree implies

$$
\operatorname{deg}\left(I-f_{n}, C, 0\right)=\operatorname{deg}\left(I-g_{n}, C, 0\right) \quad \text { for every } n \geq n_{0} .
$$

The right hand side is $1(\bmod 2)$, for $g_{n}$ is odd on $\bar{C}$, while when $n \rightarrow+\infty$ the left hand side tends to $\operatorname{Deg}(I-F, C, 0)$, a singleton set by Remark 3.6. Consequently (5.6) holds and, by Proposition 4.2, $F$ has a fixed point. This completes the proof.

\section{Acknowledgement}

This work was supported by the project 'Geometrical functional analysis in Banach spaces: variational principles and global approximation' between 
Italy and Bulgaria. The second named author thanks for the hospitality of the University Roma II, where a part of this work was done during his stay as a Visiting Professor in July 1998 and, with a fellowship of the above mentioned project, in July 1999.

\section{References}

[1] Altman, M., A fixed point theorem in Banach spaces, Bull. Acad. Polon. Sci. Cl III, 5 (1957), 89-92.

[2] Borisovitch, Y. G., Gelman, B. D., Myshkis, A. D. and Obukhovskii, V. V., Topological methods in the fixed point theory of multivalued mappings, Russian Math. Surveys, 35 (1980), 65-143.

[3] Cellina, A. and Lasota, A., A new approach to the definition of topological degree of multivalued mappings, Atti Accad. Naz. Lincei Rend. Cl. Sci. Fis. Mat. Natur., 47 (1969), 434-440.

[4] Dawidowicz, A., Méthodes homologiques dans la théorie des applications et des champs de vecteurs sphériques dans les espaces de Banach, Dissertationes Math. (Rozprawy Mat.), 326 (1993), 1-50.

[5] De Blasi, F. S., Characterizations of certain classes of semicontinuous multifunctions by continuous approximations, J. Math. Anal. Appl., 106 (1985), 1-18.

[6] De Blasi, F. S. and Myjak, J., A remark on the definition of topological degree for set-valued mappings, J. Math. Anal. Appl., 92 (1983), 445-551.

[7] Fan, Ky Fixed points and minimax theorems in locally convex spaces, Proc. Nat. Acad. Sc. U.S., 38 (1952), 121-126.

[8] Fitzpatrick, P. M. and Petryshyn, W. V., Fixed point theorems and fixed point index for multivalued mappings in cones, J. London Math. Soc. (2), 12 (1975), 75-85.

[9] Geba, K. and Granas, A., Infinite dimensional cohomology theory, J. Math. Pures Appl., 5 (1973), 147-270.

[10] Górniewicz, L., Homological methods in fixed point theory of multi-valued maps, Dissertationes Math. (Rozprawy Mat.), 129 (1976), 1-71.

[11] Górniewicz, L., Granas, A. and Kryszewski, W., On the homotopy method in the fixed point index theory of multi-valued mappings of compact absolute neighborhood retracts, J. Math. Anal. Appl., 161 (1991), 457-473.

[12] Górniewicz, L. and Lassonde, M., Approximation and fixed points for compositions of $R_{\delta}$-maps, Topology Appl., 55 (1994), 239-250.

[13] Granas, A., Sur la notion de degré topologique pour une certaine classe de transformations multivalentes dans les espaces de Banach, Bull. Acad. Polon. Sci., Série Sc. Math. Astronom. Phys., 7 (1959), 191-194.

[14] - Theorem on antipodes and theorems on fixed points for a certain class of multivalued mappings in Banach spaces, Bull. Acad. Polon. Sci., Série Sc. Math. Astronom. Phys., 7 (1959), 271-275.

[15] Hu, S. and Papageorgiou, N. S., Handbook of multivalued analysis, Vol. I, Kluwer, Dordrecht, 1997.

[16] Hukuhara, M., Sur l'application semi-continue dont la valeur est un compact convexe, Funkcial. Ekvac., 10 (1967), 43-66.

[17] Istrăţescu, V. I., Fixed point theory, Reidel, Dordrecht, 1981.

[18] Kakutani, S., A generalization of Brouwer's fixed point theorem, Duke Math. J., 8 (1941), 457-459.

[19] Lasry, J. M. and Robert, R., Analyse nonlineaire multivoque, Cahier de Mathématiques de la décision, No 7611, CEREMADE, Université de Paris IX, Dauphine, 1976.

[20] Leray, J. and Schauder, J., Topologie et équations fonctionnelles, Ann. Sci. École Norm. Sup. Sér. 3, 51 (1934), 45-78. 
[21] Lloyd, N. G., Degree theory, Cambridge University Press, Cambridge, 1978.

[22] Ma, T. W., Topological degree for set-valued compact vector fields in locally convex spaces, Dissertationes Math. (Rozprawy Mat.), 92 (1972), 1-43.

[23] Michael, E., Continuous selections I, Ann. of Math., 63 (1956), 361-382.

[24] Petryshyn, W. V. and Fitzpatrick, P. M., A degree theory, fixed point theorems and mappings theorems for multivalued noncompact mappings, Trans. Amer. Math. Soc., 194 (1974), 1-25.

[25] Rothe, E., Zur Theorie der topologischen Ordnung und der Vectorfelder in Banachshen Räumen, Compositio Math., 5 (1937), 177-197.

[26] Schwartz, J. T., Nonlinear functional analysis, Gordon and Breach, New York, 1969. 